\title{
Evaluation of Coronary Venous Anatomy by Multislice Computed Tomography
}

\author{
Jing Ping Sun ${ }^{1,2}$, Xing Sheng Yang ${ }^{1,2 *}$, Yat Yin Lam ${ }^{1}$, Mario J. Garcia ${ }^{3}$, Cheuk Man Yu ${ }^{1}$ \\ ${ }^{1}$ Division of Cardiology, S. H. Ho Cardiovascular and Stroke Centre, Prince of Wales Hospital, The Chinese University of \\ Hong Kong, Hong Kong, China \\ ${ }^{2}$ Emory University School of Medicine, Emory Hospital Midtown, Atlanta, USA \\ ${ }^{3}$ Department of Medicine, Montefiore-Einstein Heart Center, New York, USA \\ Email: *xingshengyang8@yahoo.com
}

Received July 31, 2012; revised September 10, 2012; accepted September 19, 2012

\begin{abstract}
Background: The coronary venous system is increasingly targeted for pacing in patients with severe heart failure. The recent advancement of Multi-Detector Computed Tomography (MDCT) allows accurate analysis of the coronary arteries, but little data exist on its role in assessing cardiac venous anatomy. The aim of the present study was to investigate the feasibility of using MDCT in evaluating the cardiac venous anatomy in patients with heart disease; Methods and Results: One hundred and eighteen subjects (59 \pm 11 years, 100 males) were studied by contrast enhanced 16-slice CT with retrospective ECG-reconstructions. The diameter, length, and angulations of coronary veins were measured from both volume rendered 3-dimensional images and curved multi-planar images. The coronary sinus vein was visualized in all of patients. However, the posterior, postero-lateral, lateral, antero-lateral and anterior veins were found in $71.2 \%$, $50.0 \%, 65.3 \%, 9.3 \%$ and $96 \%$ patients, respectively. Twenty-three (19.5\%) subjects had neither postero-lateral nor lateral cardiac veins. The ostial diameter angle of take-off and total length of the postero-lateral and lateral veins ranged from $1.7-7.0 \mathrm{~mm}, 38$ - 160 degrees and 2.6 - $10.6 \mathrm{~mm}$, respectively; Conclusions: This study confirms the feasibility of assessing diameter, length, and angulations of coronary veins by MDCT. This non-invasive information should be useful for pre-operative lead placement planning for patients scheduled to have cardiac resynchronization therapy.
\end{abstract}

Keywords: Cardiac Resynchronization; Coronary Vein; Multi-Detector Computed Tomography

\section{Introduction}

Multi-Detector Computer Tomography (MDCT) is a new tool for non-invasive cardiac imaging [1]. Combined with retrospective ECG-gating this new technique allows visualization of the coronary arteries with great accuracy $[1,2]$. Recent advancement can be attributed to decreased detector size, increased number of detector rows (slices), improved reconstruction algorithms and faster rotations. These improvements have lead to acquisitions with shorter scan times with higher spatial and temporal resolutions [3-6]. The coronary venous system is increasingly targeted for different electrophysiological purposes including Cardiac Resynchronization Therapy (CRT), radiofrequency catheter ablation [7], mapping [8], and defibrillation [9]. CRT, which improves outcome in patients with severe heart failure, involves the placement of pacemaker lead within the cardiac venous system $[10,11]$. Prior to placement of the pacemaker lead, patients would undergo an invasive cardiac venogram to see if they have suitable veins for CRT. Up to $10 \%-20 \%$ patients will not have suitable veins for CRT and will need to go for surgical

${ }^{*}$ Corresponding author. placement of left ventricular pacemaker lead [12-14].

The main purpose of the present study was to investigate the feasibility of assessing coronary venous system non-invasively in patients with heart disease using a retrospectively ECG-gated acquisition on a 16-slice CT scanner. Attention was focused on the availability and characteristics of coronary veins that were suitable for CRT.

\section{Methods}

\subsection{Patients}

One hundred and twenty-four subjects with known or suspected heart disease underwent ECG-gated multidetector CT scanning. Exclusions included individuals aged $<18$ years, females of childbearing age, patients with a trial fibrillation, patients with creatinine above 1.5 milligrams per deciliter or those with known contrast allergy. Our institutional review board approved the study, and all patients gave written consent.

\subsection{MDCT Procedures}

All examinations were performed on a 64-slice multi- 
detector CT scanner (Brilliance 64, Philips Medical Systems, Best, The Netherlands), using a cardiac retrospective gating technique. The CT scan was initiated following intravenous contrast agent administration (iopamidol $350,100 \mathrm{mg}$ ) after the onset of opacification of the ascending aorta. The entire heart was imaged within a single breath-hold (20 to 30 seconds). CT scan parameters included: $140 \mathrm{kVp}, 400 \mathrm{mAs}, 16 \times 0.75 \mathrm{~mm}$ collimation, $0.8 \mathrm{~mm}$ slice thickness, $0.4 \mathrm{~mm}$ slice increment, $0.42 \mathrm{sec}$ rotation time, and 0.2 to 0.3 pitch. Following the scan, the desired phase (typically between $40 \%-50 \%$ or $70 \%$ $80 \%$ ) within the R-R interval was reconstructed using the synchronized ECG signal and x-ray projection data. An adaptive multi-cycle reconstruction algorithm yielded images with temporal resolutions between 53 and $210 \mathrm{~ms}$ [15]. ECG-based dose modulation was employed in 39\% of the patients.

\subsection{Image Analysis}

Before analysis, 6 studies were excluded because the scan volume did not reveal the entire heart including the complete diaphragmatic part of the pericardium. One hundred and eighteen MDCT studies out of 124 were included in subsequent analysis. The following coronary venous segments were analyzed: the coronary sinus (CS, defined as the segment from the entrance of CS into the right atrium to the entrance of anterior cardiac vein), the mid-cardiac vein, posterior cardiac vein, postero-lateral cardiac vein, lateral cardiac vein, anterior-lateral cardiac vein, great cardiac vein (defined as the segment from the entrance of lateral cardiac vein to the entrance of anterior cardiac vein) and the anterior cardiac vein (Figure 1). The presence or absence of coronary venous segments was determined using volume-rendered images in threedimensional space. The diameter, length, and angulations of coronary veins were measured from curved multiplanar reformatted images following vessel centerline extraction (Figure 2). The length of the CS was measured from the entrance of CS into the right atrium to the entrance of anterior cardiac vein, and the diameter of the CS was measured at the orifice of coronary sinus into the right atrium. The length of coronary veins was measured from the entrance of each vein into coronary sinus vein to the end of each vein. The diameter of each coronary vein was measured at the orifice of coronary veins into the CS.

\subsection{Statistical Analysis}

Data obtained were expressed in terms of prevalence, average and standard deviation values. Microsoft Excel 2007 software was used for statistical analysis.

\section{Results}

The mean age of 118 patients (100 males, 18 females) was

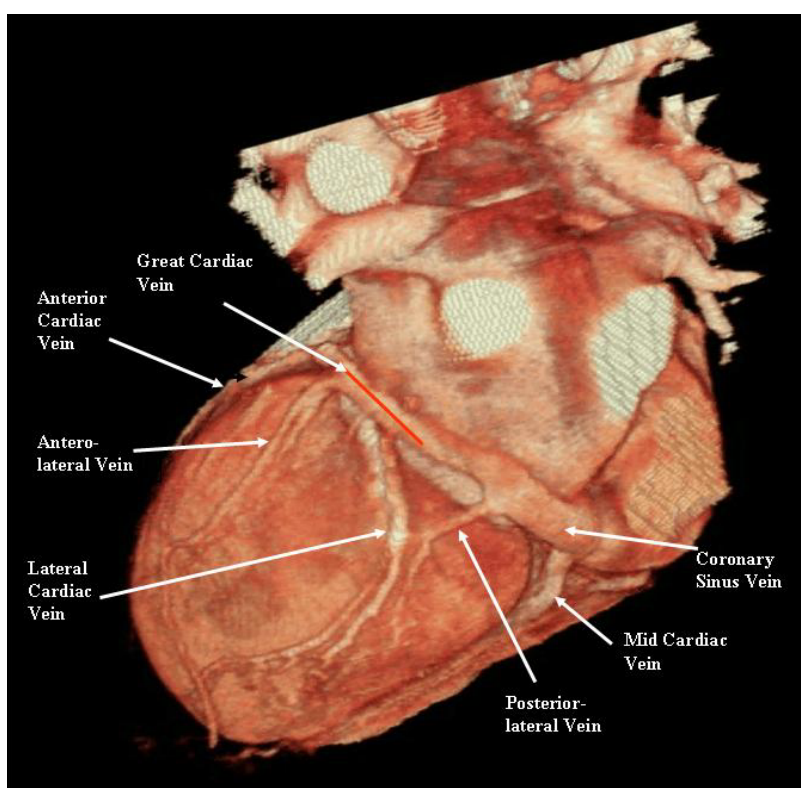

Figure 1. Coronary sinus vein and its tributaries.

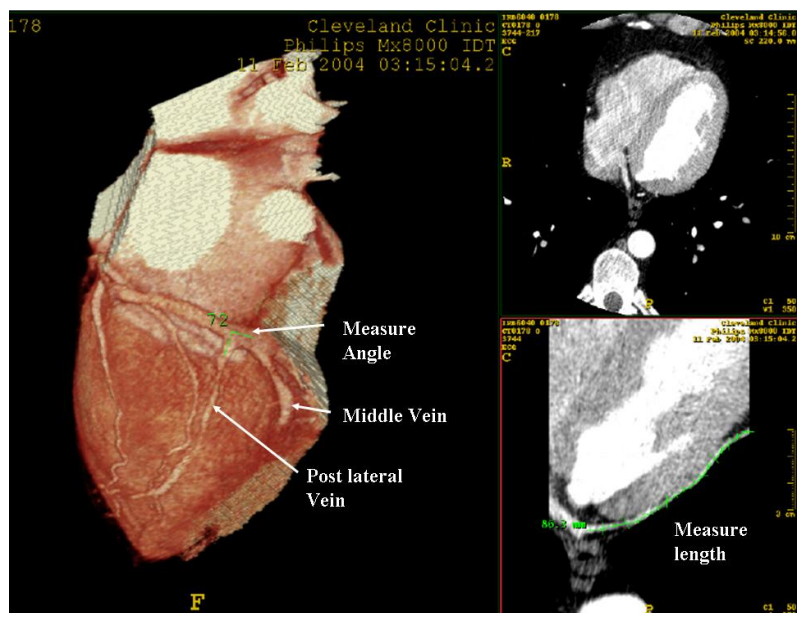

Figure 2. The diameters, lengths, and angulations of coronary veins were measured from curved multi-planar reformatted images following vessel centerline extraction.

$59 \pm 11$ years (range: 28 - 82 years). There were coronary artery disease history in 69 , valvular disease in 23 , an abnormal stress test result in 14 , heart transplantation in 7 , congestive heart failure in 3 , and hypertrophic cardiomyopathy in 2 among the 118 patients. We found a large variability of CS tree structure among the study population. The CS was visualized in all 118 patients (100\%), and the anterior vein can be seen in most of patients (96\%). The anatomical characteristics of the coronary veins from this study are listed in Tables $\mathbf{1}$ and 2. The ostial diameter, angle of take-off and total length of the posteriorlateral and lateral veins ranged from 1.7 - $7.0 \mathrm{~mm}, 38$ 160 degrees and 2.6 - $10.6 \mathrm{~mm}$, respectively.

Some patients had more than one eligible coronary veins for pacing therapy. Figure $\mathbf{3}$ shows a patient with suit- 
able lateral veins. The antero-lateral cardiac vein was visible only in $9.3 \%$ of patients; posterior-lateral cardiac vein could be detected in $50 \%$ of patients, and lateral cardiac vein was visualized in $65.3 \%$ of patients. There were 23 (19.5\%) patients who had neither a posterior-lateral nor a lateral cardiac vein. Figures 4 and 5 are 3-D reconstructed images from a patient with heart failure who underwent CS lead implantation and showed no clinical improvement after CRT. MDCT images show that the pacing lead was placed in anterior cardiac vein, as there were no other suitable cardiac veins for lead implantation in this particular patient. Current data indicate that an optimal clinical and hemodynamic response is obtained in most patients when the left ventricular electrocatheter is placed in a lateral or posterolateral position.

\section{Discussion}

In the current study, we demonstrated the feasibility of assessing cardiac venous anatomy by MDCT and we

Table 1. Characteristics of the coronary venous system.

\begin{tabular}{|c|c|c|c|c|c|c|c|c|}
\hline & & Coronary Sinus & Middle & Posterior & Postero-Lateral & Lateral & Antero-Lateral & Anterior \\
\hline \multirow{2}{*}{$\begin{array}{l}\text { Ostial Diameter } \\
\qquad(\mathrm{mm})\end{array}$} & Average & $9.9 \pm 2.4$ & $4.9 \pm 1.2$ & $3.3 \pm 1.0$ & $3.5 \pm 1.2$ & $3.2 \pm 1.1$ & $2.3 \pm 0.9$ & $3.8 \pm 0.7$ \\
\hline & Range & $5-19$ & $2-8$ & $1.4-5.7$ & $1.8-6.1$ & $1.7-7$ & $0.8-4.2$ & $2.3-5.7$ \\
\hline \multirow{2}{*}{$\begin{array}{l}\text { Length } \\
\text { (mm) }\end{array}$} & Average & $109 \pm 19$ & $60 \pm 25$ & $41 \pm 30$ & $43 \pm 30$ & $35 \pm 23$ & $23 \pm 14$ & $52 \pm 21$ \\
\hline & Range & $56-159$ & $12-118$ & $2-119$ & $2.6-106$ & $5.4-92$ & $5-51$ & $11-106$ \\
\hline \multirow{2}{*}{$\begin{array}{c}\text { Take-off } \\
\text { Angle (degree) }\end{array}$} & Average & & $72 \pm 19$ & $95 \pm 24$ & $106 \pm 26$ & $109 \pm 24$ & $83 \pm 31$ & $128 \pm 20$ \\
\hline & Range & & $35-129$ & $32-150$ & $50-160$ & $38-152$ & $25-140$ & $50-161$ \\
\hline \multicolumn{2}{|c|}{ Presence (\%) } & 100 & 94 & 71.2 & 50 & 65.3 & 9.3 & 96 \\
\hline
\end{tabular}

Table 2 . The distances between the tributary veins of coronary venous system.

\begin{tabular}{ccccc}
\hline Distance & Mid to posterior & Posterior to post-lateral & Post-lateral to lateral & Lateral to anterior \\
\hline Average $(\mathrm{mm})$ & $16 \pm 8$ & $24 \pm 11$ & $35 \pm 15$ & $43 \pm 12$ \\
Range (mm) & $3-48$ & $6-49$ & $6-64$ & $19-72$ \\
\hline
\end{tabular}

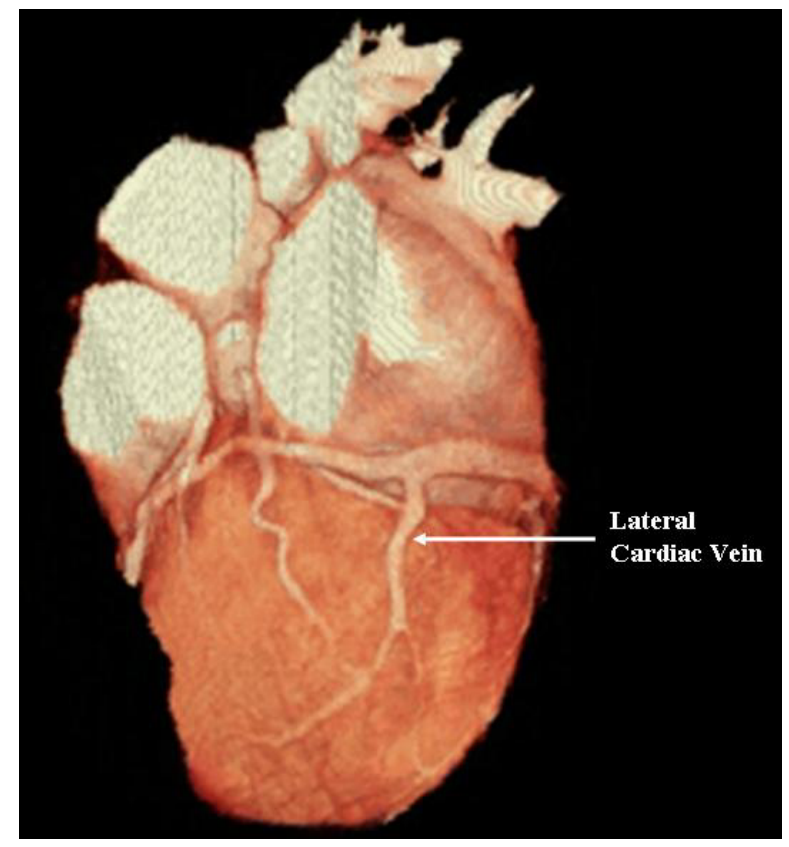

Figure 3. A patient with a suitable lateral cardiac vein for left ventricular lead placement.

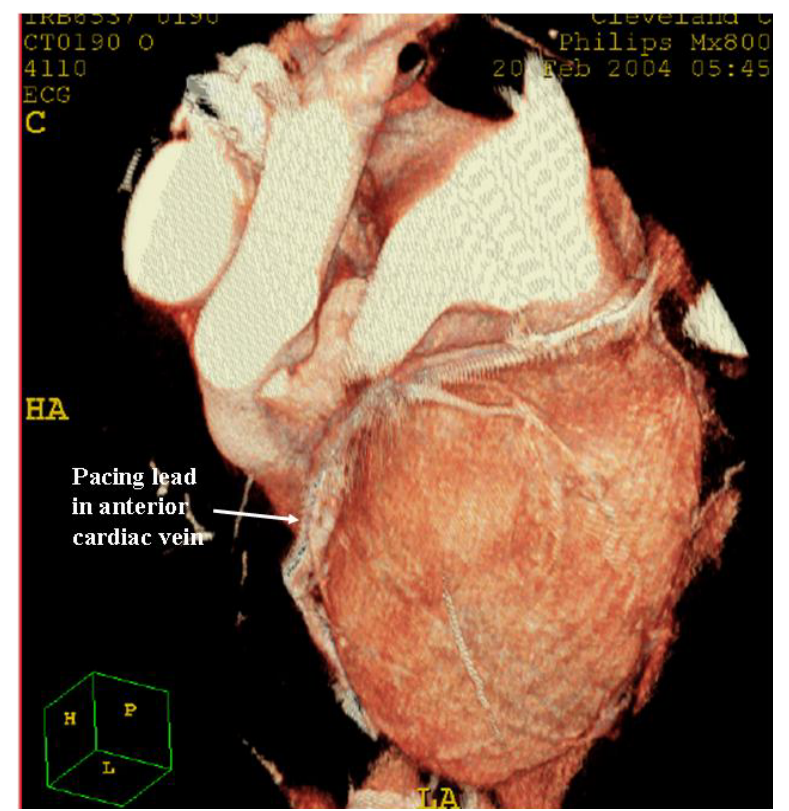

Figure 4. The image showed that the left ventricular pacing lead was positioned in anterior cardiac vein, as there was no other suitable cardiac vein. 


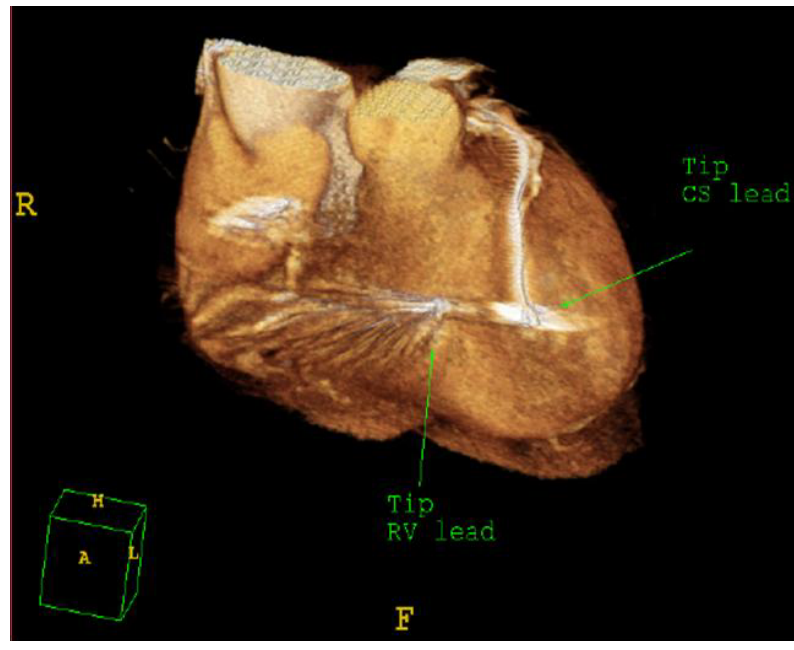

Figure 5. The image showed the close proximity of the tips of right and left ventricular leads.

found that about one-fifth of patients do not have venous anatomy suitable for optimal CRT.

Few studies have been published on cardiac vein anatomy with an emphasis on CRT planning. Two prior smaller series reported the characteristics of cardiac vein anatomy using electron beam CT [15,16]. Gerber et al. [15] studied the cardiac venous anatomy in relationship to the coronary arteries in 34 patients and Schaffler et al. [16] described the characteristics of CS and its tributary veins in 32 patients. However, the use of electron beam CT in both studies provided less spatial resolution than that observed with MDCT.

In our study, we investigated a larger group of patients than those that has previously been reported. In addition, we not only reported the appearance of coronary venous system, but also measured the angle of each venous tributary, as well as the length and diameter of each cardiac vein. Our results demonstrated there is a large variability of the coronary venous systems within the study subjects. Currently, it is thought that the positioning of left ventricular pacemaker lead in either posterior-lateral or lateral vein during CRT is associated with better clinical outcome $[17,18]$. In our study, $19.5 \%$ of the patients had neither of these veins. If our study population represents the patient population requiring CRT, one might postulate that one-fifth of the patients would benefit from going straight to surgery for epi-cardial lead placement. In the MIRACLE study [12], 8\% of the patients failed left ventricular lead implant and as successful lead placement is also determined by the size of vessel, sensing thresholds and presence of diaphragmatic pacing. In clinical practice, some patients could well have their LV lead placed in suboptimal positions, resulting in a poor clinical response after CRT. Other studies reported success rates of CRT to be $2 \%-47 \%$ and the implantation times varied from 90 minutes to 5 hours [19,20]. We believe MDCT not only provides the information regarding the presence of candidate veins, but also predict the difficulty of the lead placement procedure. Figure 5 illustrates a case in whom misplacement of pacemaker lead may have resulted in clinical response of CRT.

\section{Limitation}

This is a retrospect study, performed by contrast enhanced 16-slice CT, now are using at least 64-slice. The higher number of slices means higher resolution. The high resolution allows visualization of the entire coronary tree with extremely high accuracy and detail.

\section{Conclusion}

MDCT provides reliable non-invasive information of cardiac venous anatomy in patients. This novel information should be useful for pre-operative lead placement planning for patients scheduled to have cardiac resynchronization therapy.

\section{REFERENCES}

[1] A. F. Kopp, S. Schroeder, A. Kuettner, A. Baumbach, C. Georg, R. Kuzo, et al., "Non-Invasive Coronary Angiography with High Resolution Multidetector-Row Computed Tomography. Results in 102 Patients,” European Heart Journal, Vol. 23, No. 21, 2002, pp. 1714-1725.

[2] K. Nieman, M. Oudkerk, B. J. Rensing, P. van Ooijen, A. Munne, R. J. van Geuns, et al., "Coronary Angiography with Multi-Slice Computed Tomography,” Lancet, Vol. 357, No. 9256, 2001, pp. 599-603. doi:10.1016/S0140-6736(00)04058-7

[3] S. Achenbach, T. Giesler, D. Ropers, S. Ulzheimer, H. Derlien, C. Schulte, et al., "Detection of Coronary Artery Stenoses by Contrast-Enhanced, Retrospectively Electrocardiographically-Gated, Multislice Spiral Computed Tomography," Circulation, Vol. 103, No. 21, 2001, pp. 25352538. doi:10.1161/01.CIR.103.21.2535

[4] K. Nieman, F. Cademartiri, P. A. Lemos, R. Raaijmakers, P. M. Pattynama and P. J. de Feyter, "Reliable Noninvasive Coronary Angiography with Fast Submillimeter Multislice Spiral Computed Tomography," Circulation, Vol. 106, No. 16, 2002, pp. 2051-2054.

doi:10.1161/01.CIR.0000037222.58317.3D

[5] M. Heuschmid, A. Kuttner, T. Flohr, J. E. Wildberger, M. Lell, A. F. Kopp, et al., "Visualization of Coronary Arteries in CT as Assessed by a New 16 Slice Technology and Reduced Gantry Rotation Time: First Experiences,” Rofo, Vol. 174, No. 6, 2002, pp. 721-724. doi:10.1055/s-2002-32227

[6] T. Flohr, H. Bruder, K. Stierstorfer, J. Simon, S. Schaller and B. Ohnesorge, "New Technical Developments in Multislice CT, Part 2: Sub-Millimeter 16-Slice Scanning and Increased Gantry Rotation Speed for Cardiac Imaging,” Rofo, Vol. 174, No. 8, 2002, 1022-1027.

doi: $10.1055 / \mathrm{s}-2002-32930$ 
[7] C. Stellbrink, B. Diem, P. Schauerte, K. Ziegert and P. Hanrath, "Transcoronary Venous Radiofrequency Catheter Ablation of Ventricular Tachycardia,” Journal of Cardiovascular Electrophysiology, Vol. 8, No. 8, 1997, pp. 916-921. doi:10.1111/j.1540-8167.1997.tb00853.x

[8] R. Cappato, M. Schlüter, C. Weiss, S. Willems, T. Meinertz and K. H. Kuck, "Mapping of the Coronary Sinus and Great Cardiac Vein Using a 2-French Electrode Catheter and a Right Femoral Approach,” Journal of Cardiovascular Electrophysiology, Vol. 8, No. 4, 1997, pp. 371376. doi:10.1111/j.1540-8167.1997.tb00802.X

[9] P. R. Roberts, J. F. Urban, T. Betts, S. Allen, A. Dietz, D. E. Euler, et al., "Reduction in Defibrillation Threshold Using an Auxiliary Shock Delivered in the Middle Cardiac Vein," Pacing and Clinical Electrophysiology, Vol. 23, No. 8, 2000, pp. 1278-1282. doi:10.1111/j.1540-8159.2000.tb00944.x

[10] A. R. Leon, W. T. Abraham, S. Brozena, J. P. Daubert, W. G. Fisher, J. C. Gurley, et al., "Cardiac Resynchronization with Sequential Biventricular Pacing for the Treatment of Moderate-to-Severe Heart Failure," Journal of the American College of Cardiology, Vol. 46, No. 12, 2005, pp. 2298-2304. doi:10.1016/j.jacc.2005.08.032

[11] A. Auricchio, C. Stellbrink, C. Butter, S. Sack, J. Vogt, A. R. Misier, et al., "Pacing Therapies in Congestive Heart Failure II Study Group. Guidant Heart Failure Research Group. Clinical Efficacy of Cardiac Resynchronization Therapy Using Left Ventricular Pacing in Heart Failure Patients Stratified by Severity of Ventricular Conduction Delay," Journal of the American College of Cardiology, Vol. 42, No. 12, 2003, pp. 2109-2116. doi:10.1016/j.jacc.2003.04.003

[12] W. T. Abraham, W. G. Fisher, A. L. Smith, D. B. Delurgio, A. R. Leon, E. Loh, et al., "Cardiac Resynchronization in Chronic Heart Failure," The New England Journal of Medicine, Vol. 346, No. 24, 2002, pp. 1845-1853. doi:10.1056/NEJMoa013168

[13] D. Gras, P. Mabo, T. Tang, O. Luttikuis, R. Chatoor, A. K. Pedersen, et al., "Multisite Pacing as a Supplemental Treatment of Congestive Heart Failure: Preliminary Results of the Medtronic InSync study. Pacing and Clinical Electrophysiology, Vol. 21, No. 11, 1998, pp. 2249-2255.

\section{doi:10.1111/j.1540-8159.1998.tb01162.x}

[14] R. Manzke, M. Grass, T. Nielsen, G. Shechter and D. Hawkes, "Adaptive Temporal Resolution Optimization in Helical Cardiac Cone Beam CT Reconstruction,” Medical Physics, Vol. 30, No. 12, 2003, pp. 3072-3080. doi:10.1118/1.1624756

[15] T. C. Gerber, P. F. Sheedy, M. R. Bell, D. L. Hayes, J. A. Rumberger, T. Behrenbeck, et al., "Evaluation of the Coronary Venous System Using Electron Computed Tomography," The International Journal of Cardiovascular Imaging, Vol. 17, No. 1, 2001, pp. 65-75. doi:10.1023/A:1010692103831

[16] G. J. Schaffler, R. Groell, K. H. Peichel and R. Rienmuller, "Imaging the Coronary Venous Drainage System Using Electron-Beam CT," Surgical and Radiologic Anatomy, Vol. 22, No. 1, 2000, pp. 35-39. doi:10.1007/s00276-000-0035-1

[17] F. M. Merchant, et al., "Impact of Segmental Left Ventricle Lead Position on Cardiac Resynchronization Therapy Outcomes,” Heart Rhythm, Vol. 7, No. 5, 2010. pp. 639644.

[18] J. P. Singh, H. U. Klein, D. T. Huang, S. Reek, M. Kuniss, A. Quesada, A. Barsheshet, D. Cannom, I. Goldenberg, S. McNitt, J. P. Daubert, W. Zareba and A. J. Moss, "Left Ventricular Lead Position and Clinical Outcome in the Multicenter Automatic Defibrillator Implantation Trial Cardiac Resynchronization Therapy (MADIT-CRT) Trial," Circulation, Vol. 123, No. 11, 2011, pp. 1159-1166. doi:10.1161/CIRCULATIONAHA.110.000646

[19] S. Cazeau, C. Leclercq, T. Lavergne, S. Walker, C. Varma, C. Linde, et al., "Effects of Multisite Biventricular Pacing in Patients with Heart Failure and Intraventricular Conduction Delay," The New England Journal of Medicine, Vol. 344, No. 12, 2001, pp. 873-880. doi:10.1056/NEJM200103223441202

[20] J. H. Artrip, D. Sukerman, M. L. Dickstein and H. M. Spotnitz, "Transesophageal Echocardiography Guided Placement of a Coronary Sinus Pacing Lead," The Annals of Thoracic Surgery, Vol. 74, No. 4, 2002, pp. 1254-1256. doi:10.1016/S0003-4975(02)03788-8 